\title{
Tests for Gaussianity of the MAXIMA-1 Cosmic Microwave Background Map
}

\author{
J. H. P. Wu, ${ }^{1,2}$ A. Balbi, ${ }^{3,4,5}$ J. Borrill, ${ }^{4,6}$ P. G. Ferreira, ${ }^{7}$ S. Hanany, ${ }^{4,8}$ A. H. Jaffe,,${ }^{1,49}$ A. T. Lee, ${ }^{4,5,10}$ B. Rabii, ${ }^{4,10}$ \\ P. L. Richards, ${ }^{4,10}$ G. F. Smoot, ${ }^{4,5,9,10}$ R. Stompor, ${ }^{4,9,11}$ and C. D. Winant ${ }^{4,10}$ \\ ${ }^{1}$ Department of Astronomy, University of California, Berkeley, California 94720-3411 \\ ${ }^{2}$ Department of Physics, National Taiwan University, Taipei 106, Taiwan \\ ${ }^{3}$ Dipartimento di Fisica, Università Tor Vergata, Roma, Via della Ricerca Scientifica, I-00133, Roma, Italy \\ ${ }^{4}$ Center for Particle Astrophysics, University of California, Berkeley, California 94720-7304 \\ ${ }^{5}$ Lawrence Berkeley National Laboratory, Berkeley, California 94720 \\ ${ }^{6}$ National Energy Research Scientific Computing Center, Lawrence Berkeley National Laboratory, Berkeley, California 94720 \\ ${ }^{7}$ Astrophysics, University of Oxford, Oxford, OX1 3RH, United Kingdom \\ ${ }^{8}$ School of Physics and Astronomy, University of Minnesota/Twin Cities, Minneapolis, Minnesota 55455 \\ ${ }^{9}$ Space Sciences Laboratory, University of California, Berkeley, California 94720 \\ ${ }^{10}$ Department of Physics, University of California, Berkeley, California 94720-7300 \\ ${ }^{11}$ Copernicus Astronomical Center, Bartycka 18, 00-716 Warszawa, Poland
}

(Received 15 April 2001; published 28 November 2001)

\begin{abstract}
Gaussianity of the cosmological perturbations is one of the key predictions of standard inflation, but it is violated by other models of structure formation such as cosmic defects. We present the first test of the Gaussianity of the cosmic microwave background (CMB) on subdegree angular scales, where deviations from Gaussianity are most likely to occur. We apply the methods of moments, cumulants, the Kolmogorov test, the $\chi^{2}$ test, and Minkowski functionals in eigen, real, Wiener-filtered, and signalwhitened spaces, to the MAXIMA-1 CMB anisotropy data. We find that the data, which probe angular scales between 10 arcmin and $5 \mathrm{deg}$, are consistent with Gaussianity. These results show consistency with the standard inflation and place constraints on the existence of cosmic defects.
\end{abstract}

DOI: $10.1103 /$ PhysRevLett.87.251303

PACS numbers: 98.70.Vc, 95.85.Bh, 98.80.Es

A. Introduction.-The anisotropy in the cosmic microwave background (CMB) is arguably the cleanest cosmic signal that preserves the intrinsic statistical properties of cosmological perturbations, the energy fluctuations associated with structure formation in the universe [1]. The recent CMB observations [2-4] have unambiguously detected periodic peaks in the CMB power spectrum. The narrowness of the first peak has favored inflation [5] as the dominant mechanism for structure formation in the Universe [6], as opposed to other candidates such as cosmic defects [7]. Another key prediction of standard inflation is that the distribution of cosmic perturbations is Gaussian, while other cosmological models such as isocurvature inflation (e.g., [8]) and cosmic defects (e.g., [9]) predict otherwise. Thus tests for the Gaussianity of CMB anisotropy data can discriminate between cosmological models. In addition, a Gaussian distribution is an important ingredient in the estimation algorithm of CMB power spectra [10-12], which has been used to produce the recent MAXIMA-1 [2], BOOMERANG [3], and DASI [4] results. Adding the fact that these power spectra were further used to estimate a number of cosmological parameters to unprecedented accuracy [13-15], it is important to verify the Gaussian distribution of the CMB.

A Gaussian distribution is a consequence of a random process. Its defining property is that the higher-order (greater than two) reduced moments vanish, indicating that the mean and the two-point correlation function, or equivalently the power spectrum, contain all the statistical infor- mation in a Gaussian distribution. However, in a general map of CMB anisotropy, whose mean is zero by definition, the power spectrum carries only a small fraction of the total information. Therefore, while investigating the Gaussianity of the CMB data, this paper also examines some important remaining information (in addition to the power spectrum) which was discarded in all recently published CMB results.

Tests for the Gaussianity of CMB data have been carried out by numerous authors, mainly using data from the Differential Microwave Radiometer (DMR) on the Cosmic Background Explorer [16]. Several statistics were used including moments, cumulants, Minkowski functionals (which include genus, e.g., [17]), the three-point correlation function (e.g., [18]), bispectrum (e.g., [19,20]), wavelet transform (e.g., [21]), etc. All these tests showed that the data were consistent with Gaussianity, except for two results $[20,21]$ that may have noncosmological origins [22].

In addition to the limitations from foreground contamination and instrumental noise, the $7^{\circ}$ angular resolution of the DMR data is not ideal for tests of Gaussianity. Angular resolution is an issue because the size of the causal horizon at last scattering is about $1^{\circ}$. Thus, due to causality, there are many uncorrelated perturbations in a sky patch of superdegree size. As a result, the central limit theorem guarantees that on superdegree scales the anisotropy at last scattering will tend to be Gaussian, and may further obscure any non-Gaussianity that may be contributed from 
cosmic defects after last scattering. Therefore a CMB map with subdegree resolution can provide much more powerful tests for Gaussianity. Park et al. [23] recently used a genus test on the QMAP and Saskatoon data, which have a resolution of order of $1^{\circ}$, to show that they were consistent with Gaussianity. In this paper we report results from a series of Gaussianity tests on the MAXIMA-1 CMB map [2], which provides anisotropy information on angular scales between $10 \mathrm{arcmin}$ and $5 \mathrm{deg}$. To optimize both the resolution and the signal-to-noise ratio for these tests, we use a map with 5972 square pixels of $8^{\prime}$ each [24]. Using these high-quality data we probe for the first time the Gaussianity of CMB anisotropy on subdegree scales.

B. Karhunen-Loève transform.-We first consider the Karhunen-Loève (K-L) transform, sometimes called principal component analysis or the signal-to-noise eigenmode transformation (e.g., [11,25]). This transform enables us not only to transform the observed CMB map into uncorrelated eigenmodes of known signal-to-noise ratios but further to implement Gaussianity tests on the uncorrelated modes. For the CMB, it is standard to model the map data vector $\mathbf{d}$ as a linear sum of uncorrelated signal $\mathbf{s}$ and noise $\mathbf{n}$, with the correlation matrix $\mathbf{C} \equiv\left\langle\mathbf{d d}^{\mathrm{T}}\right\rangle=$ $\mathbf{S}+\mathbf{N}$, where $\mathbf{S}=\left\langle\mathbf{s s}^{\mathrm{T}}\right\rangle$ and $\mathbf{N}=\left\langle\mathbf{n} \mathbf{n}^{\mathrm{T}}\right\rangle$. In the noisewhitened space, $\mathbf{d}^{(\mathrm{W})}=\mathbf{N}^{-1 / 2} \mathbf{d}$, all the eigenvalues of the noise matrix, $\mathbf{N}^{-1 / 2} \mathbf{N} \mathbf{N}^{-1 / 2}=\mathbf{I}$, are simply unity. Thus the eigenvalues $\mathbf{e}^{S(\mathrm{~W})}$ of the noise-whitened signal matrix $\mathbf{N}^{-1 / 2} \mathbf{S N}^{-1 / 2}$ represent the square of signal-tonoise ratios of each eigenmode. The coefficients $\mathbf{b}^{(\mathrm{W})}$ of the noise-whitened eigenmodes in a data set can be obtained by transforming $\mathbf{d}^{(\mathrm{W})}$ to the basis which diagonalizes $\mathbf{N}^{-1 / 2} \mathbf{C N}^{-1 / 2}$. These coefficients are normally called the $\mathrm{K}-\mathrm{L}$ coefficients.

We compute $\mathbf{S}$ using the CMB power spectrum of the cosmological model best estimated from the MAXIMA-1 data [13] $\left(\Omega_{b}=0.105, \Omega_{c}=0.595, \Omega_{\Lambda}=0.3\right.$, and $h=$ 0.53 ) and including the effects introduced by the beam shape and the pixelization of the map [26]. The matrix $\mathbf{N}$ is estimated from the temporal data [24]. The resulting eigenvalues in the noise-whitened space are shown in Fig. 1, sorted in descending order. The dot-dashed line indicates that only the first 639 modes have signal-to-noise $\operatorname{ratios}\left[\mathbf{e}^{S(\mathrm{~W})}\right]^{1 / 2} \geq 1$. This number is well below our pixel number 5972. It is determined by the signal and noise levels and the observing resolution (beam) of a data set, but is independent of the pixel number of the map when the pixel size is not larger than the observing resolution.

A common technique to suppress the noise level in the map is Wiener filtering, $\mathbf{d}^{\mathrm{WF}}=\mathbf{S C}^{-1} \mathbf{d}$. This is equivalent to weighting the eigenmodes with the ratios $\mathbf{e}^{S(\mathrm{~W})} /\left(\mathbf{e}^{S(\mathrm{~W})}+1\right)$ (see Fig. 1). The sum of these ratios for all the eigenmodes is 837 , again well below the total number 5972 of the eigenmodes. We shall employ this technique in section $D$.

The K-L transform can also be used to test for Gaussianity. If the underlying map is Gaussian, then the eigenvalue-normalized K-L coefficients $\mathbf{a}^{(\mathrm{W})} \equiv \mathbf{b}^{(\mathrm{W})} /\left(\mathbf{e}^{S(\mathrm{~W})}+\right.$

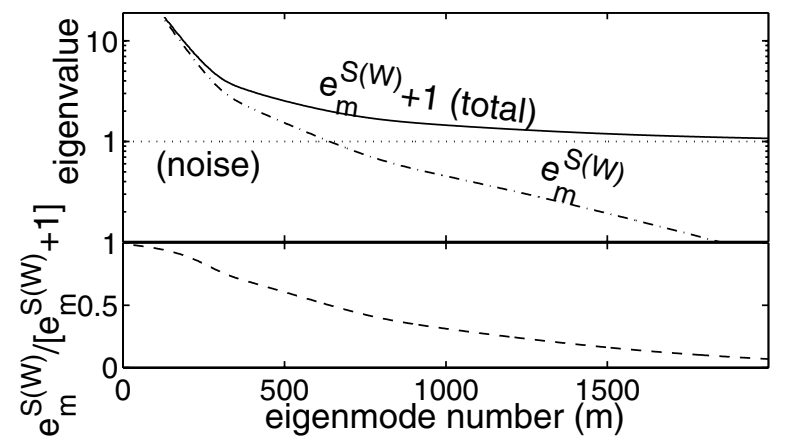

FIG. 1. Noise-whitened eigenvalues of the MAXIMA-1 CMB data (top) and the Wiener filter (bottom).

1) $)^{1 / 2}$ should be a set of Gaussian variables with mean zero and variance one. We compute the $\mathbf{a}^{(\mathrm{W})}$ of our data and then its one-point probability distribution functions (PDF) $p(\nu)$, where $\nu$ is the number of standard deviations from the mean, for both the entire 5972 and the first 639 modes (i.e., those with signal dominating over noise). Both cases easily pass the $\chi^{2}$ and the Kolmogorov tests for Gaussianity at $95 \%$ confidence.

In addition to the above tests, we also use Monte Carlo (MC) simulation to build a Gaussian reference frame for the frequentist approach, testing the null hypothesis of Gaussianity for the inflationary model that best fits the data. We generate 100000 Gaussian realizations of the MAXIMA-1 CMB map, each of which is obtained by simultaneously simulating the CMB signal and the noise $\mathbf{d}^{\mathrm{MC}}=\mathbf{C}^{1 / 2} \mathbf{g}$, where $\mathbf{g}$ is a vector of Gaussian variables of mean zero and variance one. As a first application, we use these MC maps to find the probability distribution of $p(\nu)$ at each $\nu$ for the entire 5972 and the first $639 \mathbf{a}^{(\mathrm{W})}$. Figure 2 shows that in both cases the real data lie well within the $95 \%$ confidence regions of Gaussianity (hereafter, CRG). We also compute the moments and cumulants of the first $639 \mathbf{a}^{(\mathrm{W})}$ up to tenth order, and they are all well within the $99 \%$ CRG. All these results support the conclusions not only that our map is consistent with Gaussianity but also that our estimations of noise and $\mathrm{CMB}$ power spectrum (giving $\mathbf{N}$ and $\mathbf{S}$, respectively) are consistent with the data so as to provide the proper eigenmodes for the K-L transform.

C. Minkowski functionals. - The concept of Minkowski functionals is based on integral geometry. According to Hadwiger's theorem, $\beta+1$ Minkowski functionals are sufficient to measure the morphology of a $\beta$-dimensional

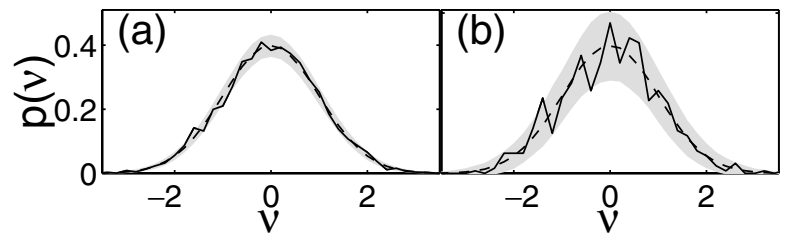

FIG. 2. PDF $p(\nu)$ of the entire 5972 (a) and the first (b) $\mathbf{a}^{(\mathrm{W})}$ of the MAXIMA-1 data (solid line), the Gaussian expectation (dashed line), and its $95 \%$ CRG (shaded region). 
pattern. In the case of $\mathrm{CMB}$ we have $\beta=2$ and thus need only three Minkowski functionals. We define the excursion set $Q$ in a given CMB field as the region in which the CMB amplitude $d$ is larger than a threshold: $Q \equiv Q(\nu)=\{d \mid(d-\mu) / \sigma>\nu\}$, where $\mu=\langle d\rangle$ and $\sigma^{2}=\left\langle d^{2}\right\rangle-\mu^{2}$. Then the surface densities $v_{i}(\nu) \equiv$ $V_{i}(Q) / A$ of Minkowski functionals $V_{i}(Q)$ for a CMB patch of angular area $A$ can be defined as

$$
\begin{gathered}
v_{0}=\frac{1}{A} \int_{Q} d A, \quad v_{1}=\frac{1}{4 A} \int_{\partial Q} d l, \\
v_{2}=\frac{1}{2 \pi} A \int_{\partial Q} \kappa d l,
\end{gathered}
$$

where $\partial Q$ is the boundary of the region $Q, d A$ and $d l$ are the differential elements of $Q$ and $\partial Q$, respectively, and $\kappa$ is the geodesic curvature of $d l$. These Minkowski functionals have different morphological meanings: $V_{0}$ is the total area of $Q, V_{1}$ is the total length of its boundary, and $V_{2}$ is the number of isolated regions (hot spots) in $Q$ minus the number of holes (cold spots). For an isotropic Gaussian field these functionals are characterized only by the variance $\sigma^{2}$ of the field and the variance of its gradient $\tau=\left\langle|\nabla d|^{2}\right\rangle / 2$, i.e., $v_{0(\mathrm{G})}=$ $\operatorname{erfc}(\nu / \sqrt{2}) / 2, \quad v_{1(\mathrm{G})}=\tau^{1 / 2} \exp \left(-\nu^{2} / 2\right) / 8 \sigma, \quad$ and $v_{2(\mathrm{G})}=\tau \nu \exp \left(-\nu^{2} / 2\right) /(2 \pi)^{3 / 2} \sigma^{2}$. We also note that the commonly used one-point $\operatorname{PDF} p(\nu)$ and genus $g$, or the "Euler-Poincaré characteristics," are simply related to the Minkowski functionals. For the twodimensional $\mathrm{CMB}$, we have $p(\nu)=-\partial V_{0} / A \partial \nu$ and $g=V_{2}+V_{0} / 2 \pi$.

We compute the $v_{0}(\nu), v_{1}(\nu)$, and $v_{2}(\nu)$ for both the entire map and the central 37\% [27] (2209 pixels, covering about $6.3^{\circ} \times 6.3^{\circ}$ ) expected to have the lowest noise. We use pixel sizes of $8^{\prime}, 16^{\prime}$, and $24^{\prime}$, the last two obtained by averaging the neighboring pixels of the original map. The results of all six cases lie within the $95 \%$ CRG obtained from the MC simulation described earlier. Figure 3 shows the results of the two cases with $8^{\prime}$ pixels. We note that while the means of the MC simulation (dashed lines) are close to the analytical isotropic Gaussian forms (dotted lines) in 3(b1)-3(b3), they deviate significantly from each other in 3(a1)-3(a3). This is due to the higher noise level near the edge of the map, contributing as an anisotropic component in the map (the rms noises per pixel in the full map and in the central part are about 162 and $57 \mu \mathrm{K}$, respectively).

We also note that some results (solid lines) appear to have systematic departure from the Gaussian expectations $\bar{v}_{i}^{\mathrm{MC}}(\nu)$ (dashed lines). For example, 3(b3) shows that the number of cold spots is less than the Gaussian expectation. To determine whether these discrepancies are really systematic, we first define

$$
I_{i}(\nu)=\int_{-\infty}^{\nu} \frac{v_{i}\left(\nu^{\prime}\right)-\bar{v}_{i}^{\mathrm{MC}}\left(\nu^{\prime}\right)}{\sigma_{i}^{\mathrm{MC}}\left(\nu^{\prime}\right)} d \nu^{\prime}, \quad i=0,1,2,
$$

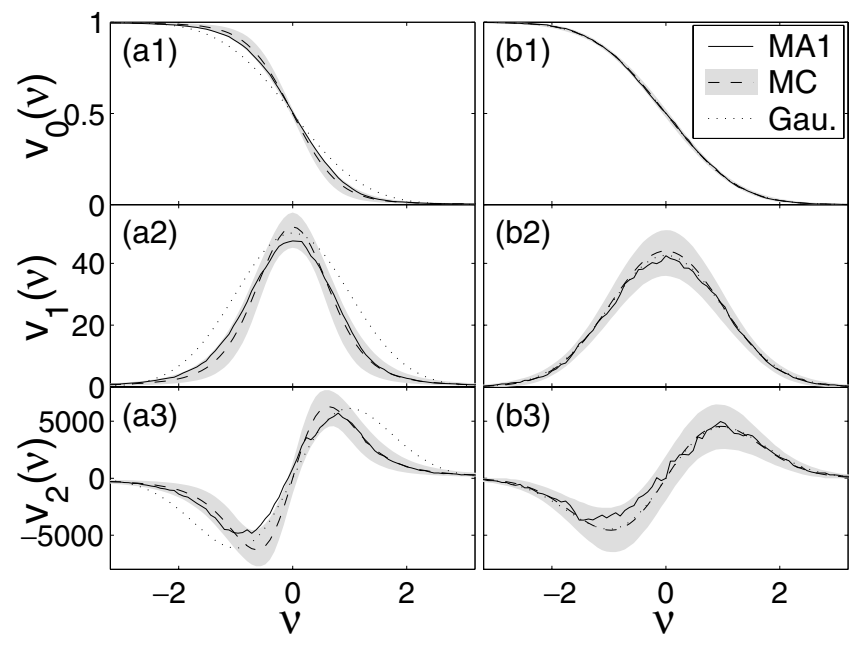

FIG. 3. Minkowski functionals (solid lines) of the entire MAXIMA-1 map (a) and the central part (b). The Gaussian expectation obtained from the MC simulation (dashed lines), its 95\% CRG (shaded regions), and the analytic Gaussian forms (dotted lines).

where $\sigma_{i}^{\mathrm{MC}}\left(\nu^{\prime}\right)$ is the standard deviation of $v_{i}\left(\nu^{\prime}\right)$ estimated from the MC simulation. Applying this to both the real data and the MC simulation, we obtain, respectively, the $\hat{I}_{i}(\nu)$ and its Gaussian-expected PDF $p\left[I_{i}(\nu)\right]$. The results from all six cases lie well within the 95\% CRG. Thus we know that the apparent systematic deviations from Gaussianity in $v_{i}$ are statistically insignificant when measured by $I_{i}(\nu)$.

D. Wiener filtering and signal-whitening techniques.-To increase the statistical significance of the CMB signal in the map, we now consider two filtering methods. One is the Wiener filtering addressed earlier, and the other is a new signal-whitening technique, $\mathbf{d}^{\mathrm{W}}=\mathbf{S}^{1 / 2} \mathbf{C}^{-1} \mathbf{d}$ [28], which not only removes the anisotropy on scales where the noise dominates (as in Wiener filtering) but also equalizes the anisotropy amplitudes on scales where the CMB signal dominates. The signal-whitening procedure will reveal in $\mathbf{d}^{\mathrm{W}}$ the features

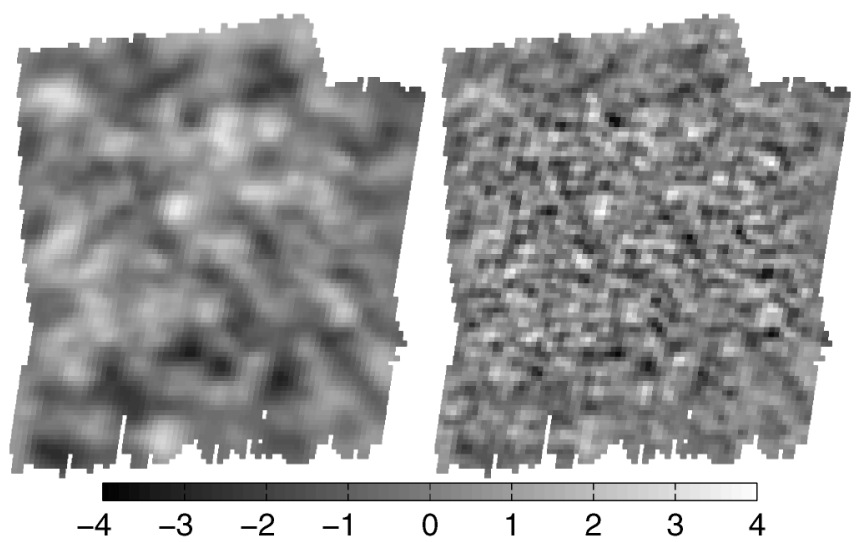

FIG. 4. The Wiener-filtered (left) and the signal-whitened (right) MAXIMA-1 map, with ranges of fluctuations of $(-3.1,3.4) \sigma$ and $(-3.7,4.3) \sigma$, respectively. 

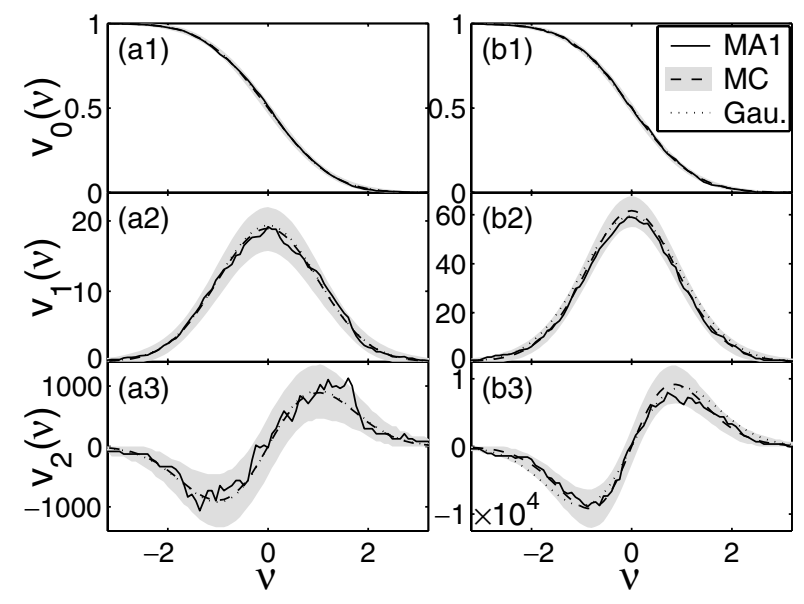

FIG. 5. Similar to Fig. 3, but for the Wiener-filtered (a) and the signal-whitened (b) MAXIMA-1 central map.

of the non-Gaussian components whose contribution in the CMB anisotropy dominates the Gaussian one within at least a range of the accessible scales [28]. We apply these filtering methods to both the real map (Fig. 4) and the MC simulation, and then compute their Minkowski functionals. We find that for both the entire map and the central part (as previously) the $v_{i}(\nu)$ and $I_{i}(\nu)$ of the filtered maps are within the $95 \%$ CRG. Figure 5 shows results of the filtered central maps. We also verify for both of the filtered maps that none of the pixels with amplitude outside the $\pm 2 \sigma$ range coincides with the locations of any known radio or infrared Astronomy Satellite point sources [29]. This was expected from the extrapolation of the amplitudes of known point sources to the frequency bands and resolution of MAXIMA-1. Thus we have no statistically significant detection of localized non-Gaussianity in our data.

E. Conclusion.-We employ moments, cumulants, the Kolmogorov test, the $\chi^{2}$ test, and Minkowski functionals in eigen, real, Wiener-filtered, and signal-whitened spaces to implement a total of 82 (not independent) hypothesis tests for Gaussianity (22 in section $B, 36$ in section $C$, 24 in section $D$ ). We show that the MAXIMA-1 CMB map is consistent with Gaussianity on angular scales between 10 arcmin and $5 \mathrm{deg}$. This gives confidence in the Gaussianity assumption used in the estimation of the CMB power spectrum [2], and consequently in the estimation of cosmological parameters $[13,15]$. The results also favor standard inflation against cosmic defects as the dominant mechanism for structure formation in the universe, as similarly concluded in the study of the recently observed CMB power spectrum. Nevertheless, the existence of cosmic defects at low energy level is still possible [6,28]. Further theoretical study of defect models will be needed to constrain this possibility in detail, and more sophisticated methods of Gaussianity tests or data with even higher resolution and signal-to-noise ratio may also be required.

J.H.P.W. and A.H.J. acknowledge support from NASA LTSA Grant No. NAG5-6552 and NSF KDI
Grant No. $9872979 . \quad$ P.G.F. acknowledges support from the Royal Society. R.S. and S.H. acknowledge support from NASA Grant No. NAG5-3941. B.R. and C. D. W. acknowledge support from NASA GSRP Grants No. S00-GSRP-032 and No. S00-GSRP-031. MAXIMA is supported by NASA Grant No. NAG5-4454 and by the NSF through the Center for Particle Astrophysics at UC Berkeley, NSF cooperative agreement AST-9120005.

[1] For a review, see, W. Hu, N. Sugiyama, and J. Silk, Nature (London) 386, 37 (1997).

[2] S. Hanany et al., Astrophys. J. 545, L5 (2000); A. T. Lee et al., astro-ph/0104459, 2001.

[3] P. de Bernardis et al., Nature (London) 404, 955, (2000); C. B. Netterfield et al., astro-ph/0104460, 2001.

[4] N. W. Halverson et al., astro-ph/0104489, 2001.

[5] A. H. Guth, Phys. Rev. D 23, 347 (1981).

[6] F. R. Bouchet et al., astro-ph/0005022, 2000; U.-L. Pen, U. Seljak, and N. Turok, Phys. Rev. Lett. 79, 1611 (1997).

[7] For a review, see A. Vilenkin and E. P. S. Shellard, Cosmic Strings and other Topological Defects (Cambridge University Press, Cambridge, UK, 1994).

[8] P. J. E. Peebles, Astrophys. J. 510, 523 (1999); P. J. E. Peebles, Astrophys. J. 510, 531 (1999).

[9] P. P. Avelino, E. P. S. Shellard, J. H. P. Wu, and B. Allen, Astrophys. J. 507, L101 (1998).

[10] M. Tegmark, Phys. Rev. D 55, 5895 (1997).

[11] J. R. Bond, A. H. Jaffe, and L. E. Knox, Phys. Rev. D 57, 2117 (1998).

[12] J. Borrill, astro-ph/9911389, 1999.

[13] A. Balbi et al., Astrophys. J. 545, L1 (2000); R. Stompor et al., astro-ph/0105062, 2001.

[14] A. E. Lange et al., Phys. Rev. D 63, 42001 (2001); P. de Bernardis et al., astro-ph/0105296, 2001.

[15] A. H. Jaffe et al., Phys. Rev. Lett. 86, 3475 (2001).

[16] G. F. Smoot et al., Astrophys. J. 396, L1 (1992).

[17] G. F. Smoot et al., Astrophys. J. 437, 1 (1994); A. Kogut et al., Astrophys. J. 464, L29, (1996); J. Schmalzing and K. M. Górski, Mon. Not. R. Astron. Soc. 297, 355 (1998).

[18] X. Luo, Phys. Rev. D 49, 3810 (1994); G. Hinshaw et al., Astrophys. J. 446, L67 (1995).

[19] A. F. Heavens, Mon. Not. R. Astron. Soc. 299, 805 (1998).

[20] P. G. Ferreira, J. Magueijo, and K. M. Gorski, Astrophys. J. 503, L1 (1998).

[21] J. Pando, D. Valls-Gabaud, and L.-Z. Fang, Phys. Rev. Lett. 81, 4568 (1998).

[22] A. J. Banday, S. Zaroubi, and K. M. Gorski, Astrophys. J. 533, 575 (2000); B. C. Bromley and M. Tegmark, Astrophys. J. 524, L79 (1999).

[23] C.-G. Park et al., Astrophys. J. 556, 582 (2001).

[24] R. Stompor et al., astro-ph/0106451 (2001).

[25] J. R. Bond, Astrophys. Lett. Commun. 32, 63 (1995); J. R. Bond, Phys. Rev. Lett. 74, 4369 (1995); M. Tegmark, A. Taylor, and A. Heavens, Astrophys. J. 480, 22 (1997); E. F. Bunn and M. White, Astrophys. J. 480, 6 (1997).

[26] J. H. P. Wu et al., Astrophys. J. Suppl. Ser. 132, 1 (2001).

[27] J.H.P. Wu et al., astro-ph/0104248, 2001.

[28] J. H. P. Wu, astro-ph/0012206, 2000.

[29] R. Paladini (private communication). 